This item was submitted to Loughborough's Research Repository by the author.

Items in Figshare are protected by copyright, with all rights reserved, unless otherwise indicated.

\title{
Comparison of gait event detection from shanks and feet in single-task and multi-task walking of healthy older adults
}

\section{PLEASE CITE THE PUBLISHED VERSION}

http://dx.doi.org/10.1109/ROBIO.2016.7866633

PUBLISHER

(C) IEEE

VERSION

AM (Accepted Manuscript)

\section{LICENCE}

CC BY-NC-ND 4.0

\section{REPOSITORY RECORD}

Kong, Weisheng, J. Lin, Lauren Waaning, Salvatore Sessa, Sarah Cosentino, Daniele Magistro, Massimiliano Zecca, Ryuta Kawashima, and Atsuo Takanishi. 2019. "Comparison of Gait Event Detection from Shanks and Feet in Single-task and Multi-task Walking of Healthy Older Adults". figshare.

https://hdl.handle.net/2134/25486. 


\title{
Comparison of Gait Event Detection from Shanks and Feet in Single-task and Multi-task Walking of Healthy Older Adults
}

\author{
W. Kong ${ }^{1}$, J. Lin ${ }^{1}$, L. Waaning ${ }^{3}$, S. Sessa ${ }^{1}$, S. Cosentino ${ }^{1}$, \\ D. Magistro ${ }^{4,5}$, M. Zecca ${ }^{3,5,6}$, R. Kawashima ${ }^{7}$, and A. Takanishi ${ }^{1,2}$
}

\begin{abstract}
Automatic and objective detection algorithms for gait events from MEMS Inertial Measurement Units data have been developed to overcome subjective inaccuracy in traditional visual observation. Their accuracy and sensitivity have been verified with healthy older adults, Parkinson's disease and spinal injured patients, using single-task gait exercises, where events are precise as the subject is focusing only on walking. Multi-task walking instead simulates a more realisitc and challenging scenario where subjects perform secondary cognitive task while walking, so it is a better benchmark. In this paper, we test two algorithms based on shank and foot angular velocity data in single-task, dual-task and multi-task walking. Results show that both algorithms fail when the subject slows extremely down or pauses due to high cognitive and attentional load, and, in particular, the first stride detection error rate of the foot-based algorithm increases. Stride time is accurate with both algorithms regardless of walking types, but the shankbased algorithm leads to an overestimation on the proportion of swing phase in one gait cycle. Increasing the number of cognitive tasks also causes this error with both algorithms.
\end{abstract}

\section{INTRODUCTION}

The quantitative and objective measurement of gait events, such as Initial Contact (IC) and Terminal Contact (TC), is important for physicians to better understand the situation of a subject, since the temporal gait parameters such as stance and swing duration, recommended [1] by the Gait and Clinical Movement Analysis Society (GCMAS), are calculated from the timing of IC and TC.

Traditional measurement via visual observation is subjective and imprecise. Therefore, high-precision motion tracking systems such as Vicon and Qualisys were introduced into some hospitals. However, high price and difficult maintenance of those systems are unaffordable for small clinics, thus limiting the number of patients benefiting form this technology. On the other hand, the rapid development of

*This research has been supported by the JSPS Grant-in-Aid for Young Scientists (Wakate B) [15K21437], FY2016 Grant Program for Promotion of International Joint Research of Waseda University, and also in part by the Program for Leading Graduate Schools, Graduate Program for Embodiment Informatics of the Ministry of Education, Culture, Sports, Science and Technology.

${ }^{1}$ Faculty of Science and Engineering, Waseda Uni., Japan

${ }^{2}$ Humanoid Robotics Institute, Waseda Uni., Japan. contactetakanishi.mech.waseda.ac.jp

${ }^{3}$ Wolfson School of Mechanical, Electrical and Manufacturing Engineering, Loughborough Uni., UK. m. zecca@lboro.ac.uk

${ }^{4}$ School of Sport, Exercise, and Health Sciences Loughborough Uni., UK.

${ }^{5}$ National Centre for Sport and Exercise Medicine, UK

${ }^{6}$ NIHR Leicester-Loughborough Diet, Lifestyle and Physical Activity Biomedical Research Unit, UK.

${ }^{7}$ Department of Functional Brain Imaging. IDAC, Tohoku Uni., Japan.
MEMS-based inertial measurement units (IMU) has turned them into a portable, affordable and reliable alternative for clinics and home-based telerehabilitation.

Many algorithms have been developed to automatically detect gait events from IMUs attached to the lower trunk [2]-[4], the shank [5]-[8] and the foot [6], [9], [10]. It is easier to detect gait events from shank and foot movement analysis, because they are closer to the ground [11].

For gait events detection from shanks, several researches [5]-[7] relied on the periodic pattern of angular velocity on sagittal plane to identify IC and TC for normal walking. First, peaks of angular velocity midswing were located, then the first local minima before and after the peaks were located and marked as TC and IC, respectively. Hundza et al. [8] detected IC and TC with this method, but they suggested to use the termination of forward swing (TOFS) instead of IC to increase the accuracy and robustness when detecting gait events for Parkinson's Disease (PD) patients.

A similar concept was used for gait events detection from feet. Jasiewicz et al. [6] located midswing at the peak of foot angular velocity and identified the first minimum before midswing as TC, while instead IC was identified at the zerocrossing point after midswing. Also Park et al. [10] identified the TC at the local minimum before midswing and the IC at the point when the signal begins to level off, close to the local minima or zero-crossing point after midswing but not easily identifiable with any of them, since they heavily denoised the angular velocity.

Foot acceleration has also been used in algorithms to detect gait events, although in general this signal is more noisy than foot angular velocity. Jasiewicz et al. [6] located IC at the peak of foot vertical acceleration around peak ankle dorsiflexion, and TC at the peak of forward-directed acceleration around peak ankle plantarflexion. Kawamura et al. [9] detected IC and TC events through the peaks of vertical acceleration jerk.

The method locating TC and IC around angular velocity peaks has gained popularity due to its simplicity, accuracy and reliability. A benchmark shank angular velocity based algorithm for PD patients [5] has a timing error of $-8.7 \pm$ $12.5 \mathrm{~ms}$ for IC and $-2.9 \pm 26.8 \mathrm{~ms}$ for TC detection; and a sensitivity of $100 \%$ for both control and PD patients during subthalamic nucleus deep brain stimulation, or $99.6 \%$ without stimulation. In a study on abnormal footfalls of patients classified as Grade D on the American Spinal Injury Association (ASIA) impairment scale [6], timing error were $-53 \pm 11 \mathrm{~ms}$ for IC and $61 \pm 10 \mathrm{~ms}$ for TC detection 
from shank angular velocity, and $-17 \pm 18 \mathrm{~ms}$ for IC and $27 \pm 28 \mathrm{~ms}$ for TC detection from foot angular velocity. Besides, the algorithms have been tested under the condition of wearing ankle orthosis and walking on snow surface [12].

To our knowledge, however, there is no study testing how these methods perform with dual-task or multi-task walking, a catogory of walking tests which requires subjects to perform at least one cognitive or motor secondary task during walking, e.g. counting. A multi-task condition provides a more realistic and challenging scenario for walking. Specifically, older adults would have more difficulties in keeping dynamic balance and have a higher risk of falling, due to the higher cognitive and attentional load required. In this scenario, a range of spatio-temporal gait parameters are affected and the variability between strides is increased [13], [14]. Multi-task walking presents challenges to automatic gait event detection, as the subject may not keep a constant walking frequency, and may slow down and even pause during walking.

Objective of this paper is, thus, to verify and compare accuracy and reliability of gait event detection methods using IMU shank and foot angular velocity data, in single-task, dual-task and multi-task walking.

\section{MATERIALS AND METHODS}

\section{A. Experiment protocol}

The experimental protocol includes the following three types of walking:

1) Single-task (ST): walking straight for 7 meters;

2) Dual-task (DT): walking while back counting by 7 from a random number between 90 and 100;

3) Multi-task (MT): walking while back counting plus holding a cup of water.

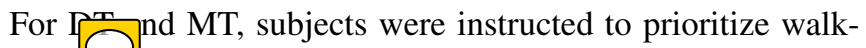
ing. $\Omega$ petitions for each exercise were executed, and the subjects were asked to start each repetition with quiet stance (QS) behind the starting line, then walk for $7 \mathrm{~m}$ and stop at the finishing line. Subjects were asked to wait at the finishing line for the instruction from the experiment assistant, without moving, while facing the direction of walking and standing on both legs.

\section{B. Experimental database}

The experimental database was recorded during a series of experimental sessions with pre-test and post-test to analyze the mobility and postural stability of older adults, and its relationship with environmental and individual factors like daily social stimulation, or physical or mental activity. 100 Japanese older adults aged over 65 , mentally and physically healthy, were recruited and participated in the experiment at the Institute of Development, Aging and Cancer, Tohoku University, Japan. The experiment protocol was approved by the ethical committees of Waseda University and Tohoku University and all participants provided written informed consent. Anthropometric data of the participants are shown in Table I. In this study, we tested gait events detection algorithm on the trials of ST, DT, and MT walking patterns of 20 subjects randomly extracted from the database.

TABLE I

ANTHROPOMETRIC INFORMATION OF EXPERIMENTAL SUBJECTS

\begin{tabular}{lllll}
\hline Gender & $\mathrm{N}$ & Age & Weight & Height \\
\hline Female & 60 & $68.4 \pm 3.2$ & $51.8 \pm 7.2$ & $152.4 \pm 4.8$ \\
Male & 40 & $69.3 \pm 3.3$ & $65.2 \pm 9.1$ & $165.0 \pm 6.6$ \\
All & 100 & $68.8 \pm 3.2$ & $57.1 \pm 10.4$ & $157.4 \pm 8.3$ \\
\hline
\end{tabular}

\section{Data recording system}

The miniaturized $(17 \times 20 \times 8 m m ; 3.9 g) \quad$ IMU, named Waseda Bioinstrumentation 4R (WB-4R) [15], was used for this study (Fig. 1). The WB-4R sensor consists of triaxial MEMS-based gyroscope (LYPR540AH, $\pm 400 / \pm$ $1600 \mathrm{dps}$ ), accelerometer (LIS331DLH, $\pm 8 \mathrm{G}$ ), and magnetometer (HMC5843, \pm 4 Gauss), measuring respectively the angular velocity, acceleration and magnetic field. IMU sensors were secured to the body with tight elastic bands.

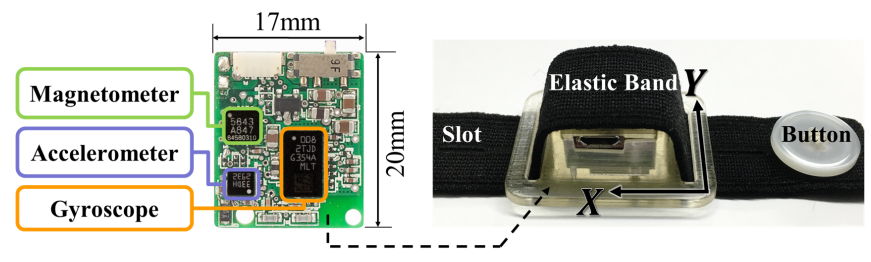

Fig. 1. WB-4R IMU and elastic band for sensor placement.

Nine synchronized IMUs acquired data at $200 \mathrm{~Hz}$ and sent them via Bluetooth to a personal computer for data logging. In this study, only gyroscope data from both shanks and feet are used for gait event detection. Gyroscope data from the chest is also used to exclude the strides for turning back after the subject reaches the goal. The axes of the IMUs are calibrated by software to have the $\mathrm{x}$-axis pointing to the front, $y$-axis pointing to the right of the body, and z-axis pointing down (Fig. 2).

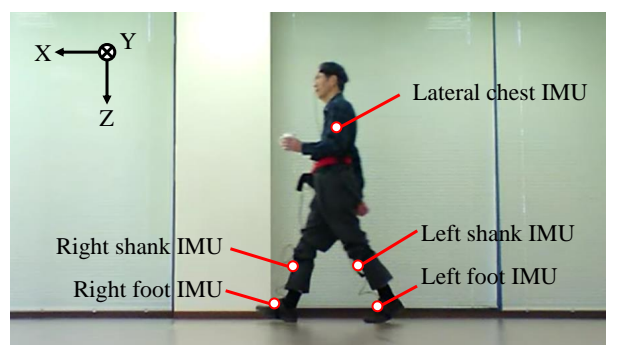

Fig. 2. Sensor placement. In this research only data from sensors on both shanks and feet were used for gait events detection, while data from the chest IMU was used to detect turning at the end of the experiment.

Two high resolution digital web cameras $(1920 \times 1080$, $20 \mathrm{fps}$ ) were placed on the front and on the side of the experimental area, to allow and facilitate manual segmentation from video for control purposes. 


\section{Walking period segmentation}

The target of walking period segmentation is to exclude data before and after the experiment, including the strides for turning back after the end line is reached. The segmentation algorithm uses angular velocity from both shanks and chest as follows:

first, the magnitude of angular velocity on sagittal plane is calculated and averaged between left and right shanks,

$$
\boldsymbol{\Omega}_{s}=\frac{\left|\omega_{l s, y}\right|+\left|\omega_{r s, y}\right|}{2}
$$

where $\omega_{l s, y}$ and $\omega_{r s, y}$ are the angular velocities around $\mathrm{Y}$ axis (medial-lateral axis) for the left and right shank respectively. Absolute values and average between shanks are used to smooth the signal during the walking period and make it as continuous as possible, avoiding the entire walking period to be divided into several short periods.

Then, a threshold at $0.5 \mathrm{rad} / \mathrm{s}$ (about $30 \mathrm{deg} / \mathrm{s}$ ) is applied to assess the movement of both shanks. The value of the threshold is selected on empirical basis.

$$
b_{i}= \begin{cases}1, & \Omega_{s, i}>0.5 \mathrm{rad} / \mathrm{s} \\ 0, & \text { otherwise }\end{cases}
$$

Similarly, the turn at the end of the experiment is excluded by applying $0.5 \mathrm{rad} / \mathrm{s}$ threshold on the low pass filtered (5th order, $1 \mathrm{~Hz}$ cutoff Butterworth filter) chest angular velocity on the transverse plane $\left(\omega_{c, z}^{\prime}\right.$ in Equation 3), with 0 and 1 representing turning and non-turning respectively.

$$
m_{i}= \begin{cases}0, & \left|\omega_{c, z}^{\prime}\right| \geq 0.5 \mathrm{rad} / \mathrm{s} \\ 1, & \text { otherwise }\end{cases}
$$

Fast turns in less than $500 \mathrm{~ms}$ (100 samples) can happen during walking, and are removed by morphological closure,

$$
\mathbf{M}=\mathbf{m} \bullet \mathbf{1}_{1 \times 100}=\left(\mathbf{m} \oplus \mathbf{1}_{1 \times 100}\right) \ominus \mathbf{1}_{1 \times 100}
$$

where $\bullet, \oplus$ and $\ominus$ represent morphological closure, dilation and erosion, respectively. The non-turning mask $\mathbf{M}$ is then applied on $\mathbf{b}$ to remove the turn from the walking phase segmentation.

$$
\mathbf{b}^{\prime}=\mathbf{b} \wedge \mathbf{M}
$$

Finally, walking periods shorter than 200 samples (about $1 s$ ) are merged by using morphological closure, to remove pauses during walking, and the longest continuous period measured after merging is selected as the walking period.

$$
\mathbf{B}=\mathbf{b}^{\prime} \bullet \mathbf{1}_{1 \times 200}
$$

After auto-segmentation, a quick manual check was conducted to find problematic segmentations, by screening graphs similar to Fig. 3 and the corresponding video. The ones without walking, or with sensor error (extremely high noise, wrong offset, or large range) were removed. For graphs containing multiple walking parts, with unclear start or stop of walking (e.g. pause in the 1 st stride, or when a subject stepped forward and backward right before the "Start" instruction), and with turns in the middle of walking, the walking period was manually modified.

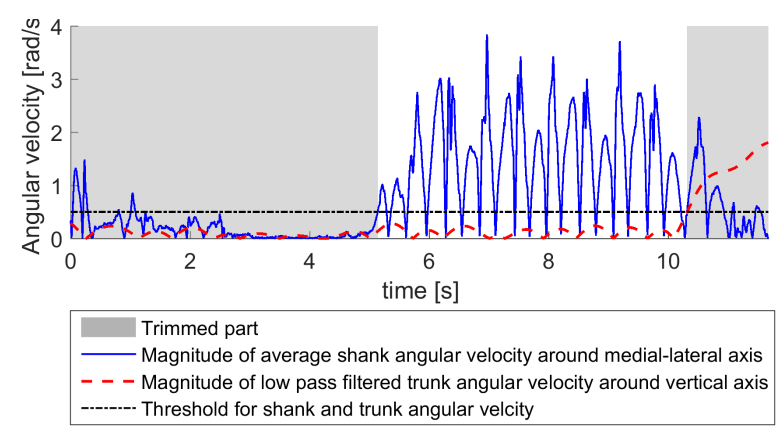

Fig. 3. Walking period segmentation. Movements before "Start" and the final turn after the test were trimmed.

\section{E. Step event detection}

Two gait event detection algorithms to find IC and TC for each leg were developed, using respectively the angular velocities of shanks and feet.

The algorithm to detect IC and TC from shank data is based on [5], and follows these steps:

1) Apply 10th order $30 \mathrm{~Hz}$ cutoff frequency Butterworth low pass filter (LPF) on the shank angular velocity around medial-lateral (Y) axis.

$$
\omega_{y}^{\prime}=\operatorname{LPF}\left(\omega_{s, y}\right)
$$

2) Trim the data outside the walking period segmented in Section II-D.

$$
\boldsymbol{\Omega}=\left\{\omega_{y, i}^{\prime}: N_{0} \leq i \leq N_{1}\right\}
$$

where $N_{0}$ and $N_{1}$ are the start and stop frame numbers of the walking period. After trimming, the new data length is $L=N_{1}-N_{0}+1$.

$$
B_{i}= \begin{cases}1, & N_{0} \leq i \leq N_{1} \\ 0, & \text { otherwise }\end{cases}
$$

3) Find fast swing part by setting threshold at $1 \mathrm{rad} / \mathrm{s}$.

$$
s_{i}= \begin{cases}1, & \Omega_{i}>1 \mathrm{rad} / \mathrm{s} \\ 0, & \text { otherwise }\end{cases}
$$

The swing part shorter than $100 \mathrm{~ms}$ is removed by morphological opening.

$$
\mathbf{S}=\mathbf{s} \circ \mathbf{1}_{1 \times 20}
$$

4) Find the positive edges (pe) and negative edges (ne) of the swing part, and set $250 \mathrm{~ms}$ search window before each positive edge and after each negative edge.

$$
\begin{aligned}
& \mathbf{S}^{\prime}=[0, \mathbf{S}, 0] \\
& \mathbf{p e}=\left\{i: S_{i+1}^{\prime}-S_{i}^{\prime}=1\right\} \\
& \mathbf{n e}=\left\{i: S_{i+2}^{\prime}-S_{i+1}^{\prime}=-1\right\}
\end{aligned}
$$

5) Set the minimum within the search window before positive edge as TC.

$$
T C_{k}=\underset{\left(p e_{k}-50\right) \leq i<p e_{k}}{\arg \min }\left(\Omega_{i}\right)
$$

where $k$ represents the $k^{\text {th }}$ stride during $7 \mathrm{~m}$ walking. 
6) Set the first minima under threshold $-0.5 \mathrm{rad} / \mathrm{s}$ within search window after negative edge as IC.

$$
\begin{aligned}
I C_{k}= & \min \left(\left\{i \mid n e_{k}<i \leq\left(n e_{k}+50\right):\right.\right. \\
& \left(\Omega_{i}<\Omega_{i-1}\right) \wedge\left(\Omega_{i} \leq \Omega_{i+1}\right) \wedge \\
& \left.\left.\left(\Omega_{i}<-0.5 \mathrm{rad} / \mathrm{s}\right)\right\}\right)
\end{aligned}
$$

The algorithm to detect IC and TC from foot data is based on [6]. It is similar to the shank-based algorithm, but in the 6th step the IC is identified at the first cross zero point after midswing, instead of the first minima:

6) Set the first cross zero point within search window after negative edge as IC.

$$
\begin{aligned}
I C_{k}= & \min \left(\left\{i \mid n e_{k}<i \leq\left(n e_{k}+50\right):\right.\right. \\
& \left.\left.\Omega_{i-1}>0 \geq \Omega_{i}\right\}\right)
\end{aligned}
$$

The rest part of the foot-based algorithm is the same as the shank-based algorithm, except replacing $\omega_{s, y}$ in Equation 7 with $\omega_{f, y}$, the Y component of the foot angular velocity.

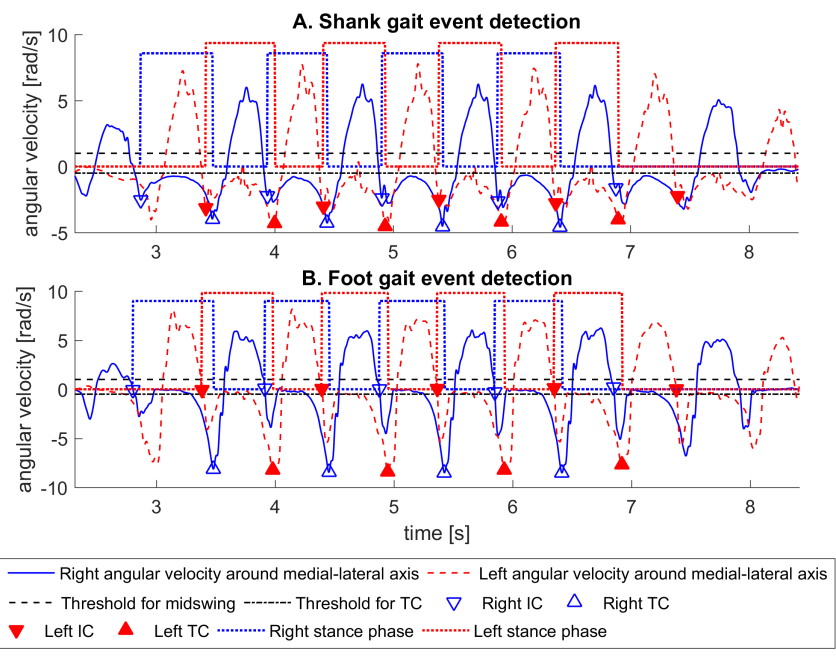

Fig. 4. Example of gait event detection with shank and foot angular velocity, with first and last stride of each leg excluded.

Once ICs and TCs are found, they are used to divide strides. One stride is defined as starting from IC, following the stance phase and then swing phase, and finally finished with the IC of the next stride. The ICs from left and right legs are used to define the left and right stride, respectively.

Also, ICs and TCs are used to calculate the temporal parameters, i.e. stride time length, stance phase length, swing phase length, and double stance phase length. The first stride starts from and last stride ends with quiet stance, therefore are removed from the computation of temporal parameters.

\section{F. Manual comparison}

Manual segmentation was done by playing the video recorded from the lateral camera, frame by frame to find the IC and TC. The video is taken at 20 FPS so the error of manual segmentation is $\pm 50 \mathrm{~ms}$. Stride time length, stance phase length, swing phase length, and double stance phase length were also calculated from manual gait detection, whose error is $\pm 100 \mathrm{~ms}$. The IC, TC and gait temporal parameters of each leg are compared with the result of automatic gait event detection and the results is shown in the following section.

\section{RESULTS}

Concerning the sensitivity (TPR) and positive predictive value (PPV) of gait events detection, we found that the algorithm based on foot data bears a much higher rate of detection error compared with detection from shank data, see Table II.

TABLE II

TPR AND PPV OF GAIT EVENTS DETECTION

\begin{tabular}{llll}
\hline & Single task & Dual task & Triple task \\
\hline Shank TPR & $100.0 \%$ & $99.4 \%$ & $100.0 \%$ \\
Foot TPR & $99.4 \%$ & $97.7 \%$ & $99.0 \%$ \\
\hline Shank PPV & $100.0 \%$ & $100.0 \%$ & $100.0 \%$ \\
Foot PPV & $100.0 \%$ & $97.7 \%$ & $100.0 \%$ \\
\hline Number of strides & 161 & 174 & 197 \\
\hline
\end{tabular}

Errors can be categorized into: unclear first stride, extremely slow walking speed, and pause in midswing.

Unclear first stride happens when the 1st stride is slow and short with the angular velocity over $1 \mathrm{rad} / \mathrm{s}$ shorter than $100 \mathrm{~ms}$ (false negative), or when the subject moves one leg right before the experiment (false positive). Although in this study the first and last stride are excluded from the computation of temporal gait parameters, the error in detection does affect the alignment between auto and manual segmentation.

The second type of error is caused by extremely slow walking speed, as shown in Fig. 5. The IC after the midswing between 3 and $4 s$ is missing, because the subject slowed down the walking speed, considering the next answer for counting, and took $400 \mathrm{~ms}$ ( $150 \mathrm{~ms}$ outside the $250 \mathrm{~ms}$ search window) to finally put the foot on the ground.

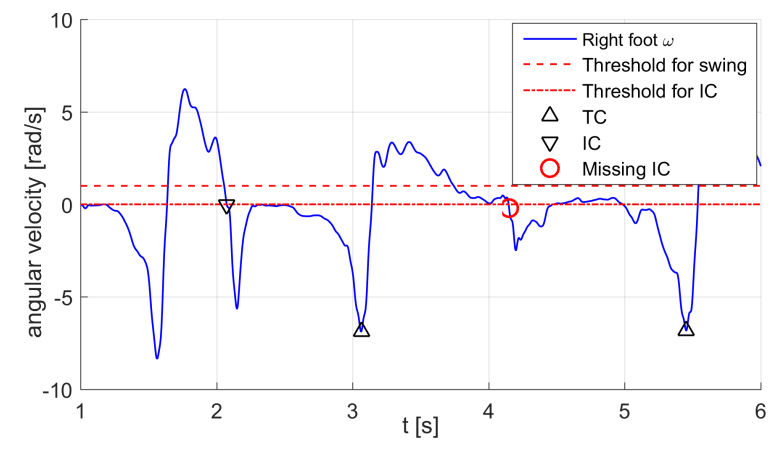

Fig. 5. Failure caused by low walking speed.

The last error type comes from pause in midswing. As shown in Fig. 6, there are two continuous major peaks between 5 and $6.5 \mathrm{~s}$. After checking the video, we understand that they belong to one single stride because the subject paused in midswing without putting the swing leg down to the ground. Since the first peak is not recognized by the 
algorithm as swing phase, the pause in midswing is instead regarded as TC. In this specific case, the pausing causes TC timing error but not error in stride detection. However, it can easily result in error when the subject rests the paused swing leg on the ground.

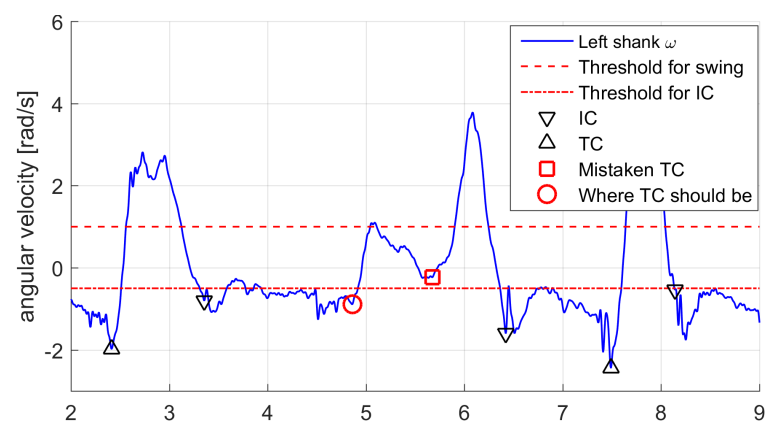

Fig. 6. Error and potential failure caused by pausing in midswing.

Concerning the accuracy, the timing of automatically detected TC and IC are highly correlated with the manual segmentation, regardless of the sensor position and the number of tasks during walking. However we found that the sampling frequency of the web cam differs from trial to trial (Fig. 7), which causes the overall correlation coefficient to be significantly low compared with the average of single trials. Besides, although for both IC and TC the averaged $R^{2}$ is over $0.99, R^{2}$ of TC is relatively lower than IC. This difference is marginal for single-task ( $p$ in T-test less than 0.2 ), but is significant for dual and triple tasks, with $p$ less than 0.005 and 0.001 respectively.

TABLE III

$R^{2}$ OF IC AND TC (MEAN \pm STD FOR EACH TRIAL)

\begin{tabular}{llll}
\hline & Single task & Dual task & Triple task \\
\hline Shank IC & $0.9997 \pm 0.0003$ & $0.9996 \pm 0.0003$ & $0.9997 \pm 0.0004$ \\
Foot IC & $0.9997 \pm 0.0003$ & $0.9996 \pm 0.0003$ & $0.9997 \pm 0.0003$ \\
\hline Shank TC & $0.9963 \pm 0.0112$ & $0.9985 \pm 0.0014$ & $0.9979 \pm 0.0019$ \\
Foot TC & $0.9968 \pm 0.0096$ & $0.9988 \pm 0.0006$ & $0.9979 \pm 0.0018$ \\
\hline
\end{tabular}

For stride time the error, defined as $t_{\text {auto }}-t_{\text {manual }}$, distributes around zero and within the error range of manual segmentation $( \pm 100 \mathrm{~ms})$. Fig. 8 shows the error in stride time, where the horizontal axis represents the segments used for gait detection (S: shank; F: foot), as well as the types of test (1: single-task; 2: dual tasks; 3 : triple tasks). No difference is found between shank and foot, and between the single and multiple tasks.

However, for the duration of stance phase and double stance phase, the timing error of both shank-based and footbased algorithm increases with the number of tasks in the negative direction, leading to an underestimation on the duration of stance phase. Moreover, the absolute timing error of shank-based algorithm is significantly larger than the footbased algorithm. For example in Fig. 9 and 10, for S1 the absolute median error is over $60 \mathrm{~ms}$ while for $\mathrm{F} 1$ the error is around and even smaller than $5 \mathrm{~ms}$.

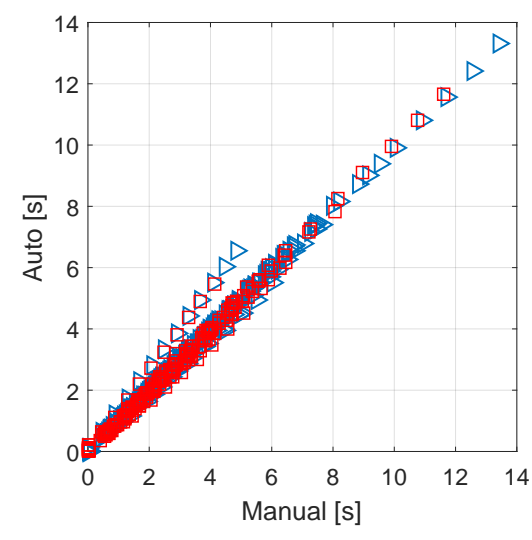

$\triangleright$ IC after reset

$\square \quad$ TC after reset

Fig. 7. Example of drifted difference between manual and auto gait events detection due to unstable web cam sampling frequency. Both IC and TC are reset by setting the new original point at $\left[t_{m 1}, \hat{t}_{a 1}\right]$, where $t_{m 1}$ is the manually segmented timing of first IC/TC, while $\hat{t}_{a 1}$ is the auto segmented timing estimated by linear regression of each trial.

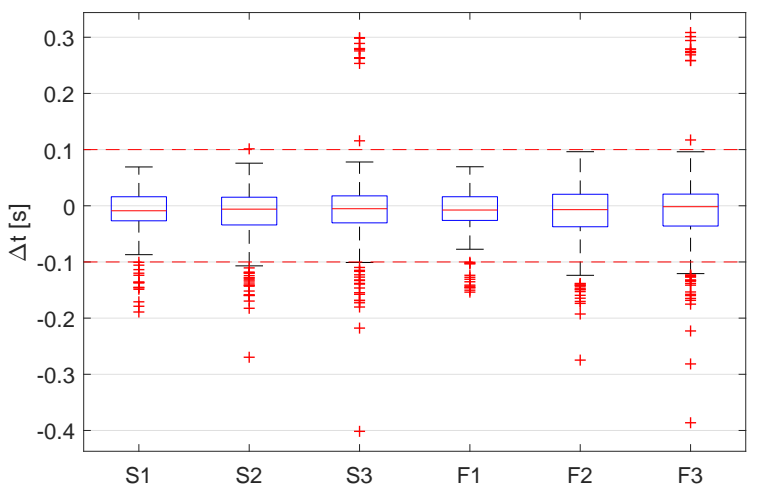

Fig. 8. Error in stride time (auto - manual). The error range for manual segmentation is marked with red dash lines.

The similar results are found for swing phase, though the error is in the opposite direction with stance phase and double stance phase: either increasing the number of tasks or detecting from shanks instead of feet causes overestimation on the duration of swing phase, see Fig. 11.

\section{CONCLUSION AND FUTURE WORKS}

In this work, we tested two independent gait event detection algorithms, one based on shank angular velocity and one based on foot angular velocity, under the conditions of 7 meter single-task walking, dual-task walking (backcounting), and multi-task walking (backcounting with holding a cup of water). Automatic walking segmentation was implemented to automatically trim data before the start and at the end of the walking test when subject turns back.

The results show that both algorithms fail when the subject suddenly slows down or pause during midswing, and the error rate of the foot-based algorithm is much higher, especially in the detection of the first stride. Besides, although both algorithms are accurate on stride time regardless of the walking type, when estimating the duration of stance phase, swing phase and double stance phase, we found that the shank-based algorithm bears a larger bias compared to 


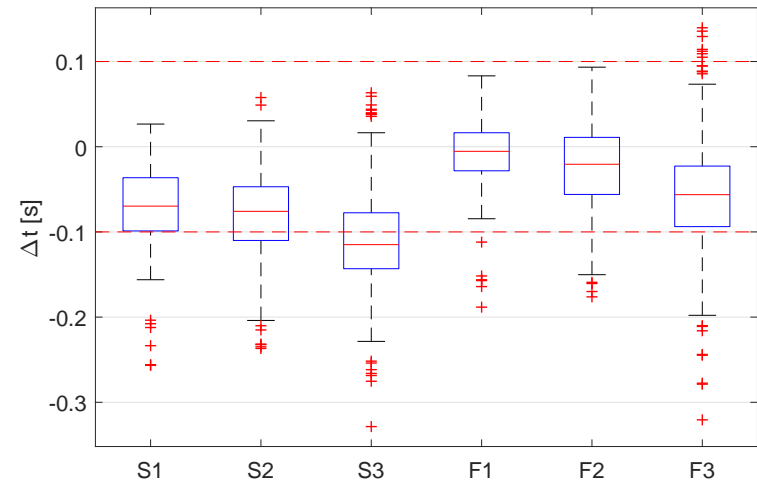

Fig. 9. Error in stance phase duration (auto - manual).

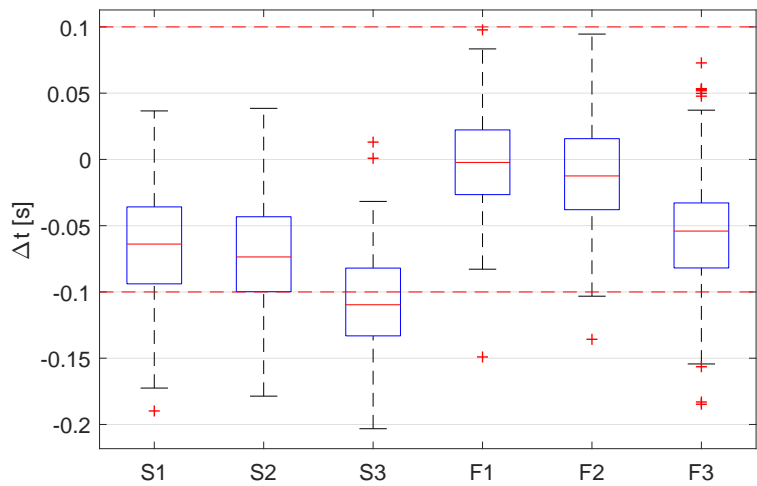

Fig. 10. Error in double stance phase duration (auto - manual).

the foot-based one. Moreover, increasing the number of cognitive tasks leads to a larger bias for both algorithms, overestimating the swing phase time and underestimating the stance and double stance phase duration.

In the future, we will improve both algorithms to increase the gait events detection rate during multi-task walking, detect the cause of and solve the bias in the duration of swing phase, stance phase and double stance phase.

\section{ACKNOWLEDGMENT}

The authors would like to express their thanks to the Italian Ministry of Foreign Affairs, General Directorate

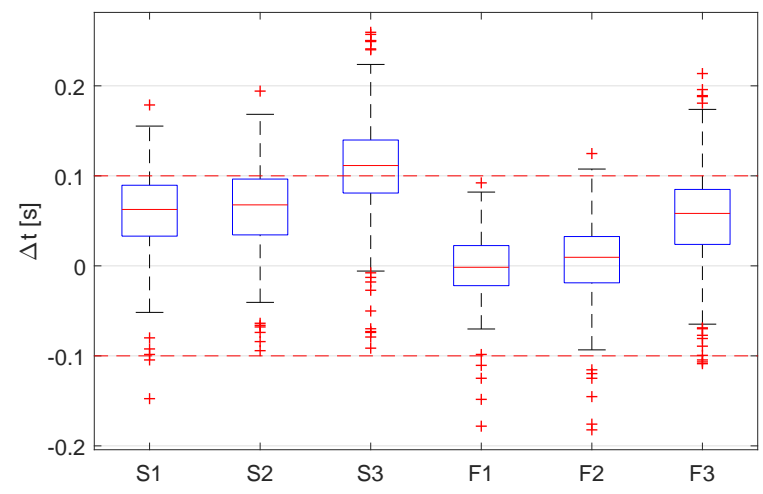

Fig. 11. Error in swing phase duration (auto - manual). for Cultural Promotion and Cooperation for its support to RoboCasa; Waseda University Program for Leading Graduate Schools; Tokyo Women's Medical University/Waseda University Joint Institution for Advanced Biomedical Sciences (TWIns); JSPS Postdoctoral Fellowship Program for Foreigner Researchers FY2012. The authors would also like to express their gratitude to STMicroelectronics, Life Performance Research, SolidWorks Corp for their support.

\section{REFERENCES}

[1] T. Öberg, A. Karsznia, and K. Öberg, "Basic gait parameters: reference data for normal subjects, 10-79 years of age," Journal of rehabilitation research and development, vol. 30, pp. 210-210, 1993.

[2] W. Zijlstra and A. L. Hof, "Assessment of spatio-temporal gait parameters from trunk accelerations during human walking," Gait \& posture, vol. 18, no. 2, pp. 1-10, 2003.

[3] J. McCamley, M. Donati, E. Grimpampi, and C. Mazzà, "An enhanced estimate of initial contact and final contact instants of time using lower trunk inertial sensor data," Gait \& posture, vol. 36, no. 2, pp. 316-318, 2012.

[4] D. Trojaniello, A. Cereatti, and U. Della Croce, "Accuracy, sensitivity and robustness of five different methods for the estimation of gait temporal parameters using a single inertial sensor mounted on the lower trunk," Gait \& posture, vol. 40, no. 4, pp. 487-492, 2014.

[5] A. Salarian, H. Russmann, F. J. Vingerhoets, C. Dehollain, Y. Blanc, P. R. Burkhard, and K. Aminian, "Gait assessment in parkinson's disease: toward an ambulatory system for long-term monitoring," Biomedical Engineering, IEEE Transactions on, vol. 51, no. 8, pp. 1434-1443, 2004.

[6] J. M. Jasiewicz, J. H. Allum, J. W. Middleton, A. Barriskill, P. Condie, B. Purcell, and R. C. T. Li, "Gait event detection using linear accelerometers or angular velocity transducers in able-bodied and spinalcord injured individuals," Gait \& Posture, vol. 24, no. 4, pp. 502-509, 2006.

[7] S. Yang, J.-T. Zhang, A. C. Novak, B. Brouwer, and Q. Li, "Estimation of spatio-temporal parameters for post-stroke hemiparetic gait using inertial sensors," Gait \& posture, vol. 37, no. 3, pp. 354-358, 2013.

[8] S. R. Hundza, W. R. Hook, C. R. Harris, S. V. Mahajan, P. A. Leslie, C. A. Spani, L. G. Spalteholz, B. J. Birch, D. T. Commandeur, and N. J. Livingston, "Accurate and reliable gait cycle detection in parkinson's disease," IEEE Transactions on Neural Systems and Rehabilitation Engineering, vol. 22, no. 1, pp. 127-137, 2014.

[9] K. Kawamura, Y. Morita, J. Okamoto, K. Saito, S. Sessa, M. Zecca, A. Takanishi, M. Fujie, et al., "Gait phase detection using foot acceleration for estimating ground reaction force in long distance gait rehabilitation," Journal of Robotics and Mechatronics, vol. 24, no. 5, pp. 828-837, 2012.

[10] M.-H. Park, K.-Y. Kwak, and D.-W. Kim, "Developing a portable gait cycle detection system using an inertial sensor and evaluating the accuracy of the gait cycle detection," Technology and Health Care, vol. 24, no. s1, pp. S69-S76, 2015.

[11] D. Trojaniello, A. Cereatti, E. Pelosin, L. Avanzino, A. Mirelman, J. M. Hausdorff, and U. Della Croce, "Estimation of step-by-step spatio-temporal parameters of normal and impaired gait using shankmounted magneto-inertial sensors: application to elderly, hemiparetic, parkinsonian and choreic gait," Journal of neuroengineering and rehabilitation, vol. 11, no. 1, p. 1, 2014.

[12] S. Sessa, M. Zecca, L. Bartolomeo, T. Takashima, H. Fujimoto, and A. Takanishi, "Reliability of the step phase detection using inertial measurement units: pilot study," Healthcare Technology Letters, vol. 2, no. 2, pp. 58-63, 2015.

[13] P. R. Brustio, D. Magistro, E. Rabaglietti, and M. E. Liubicich, "Agerelated differences in dual task performance: A cross-sectional study on women," Geriatrics \& gerontology international, 2016.

[14] L. Lundin-Olsson, L. Nyberg, Y. Gustafson, et al., "Stops walking when talking as a predictor of falls in elderly people," Lancet, vol. 349, no. 9052, p. 617, 1997.

[15] W. Kong, S. Sessa, S. Cosentino, M. Zecca, K. Saito, C. Wang, U. Imtiaz, Z. Lin, L. Bartolomeo, H. Ishii, et al., "Development of a real-time imu-based motion capture system for gait rehabilitation," in Robotics and Biomimetics (ROBIO), 2013 IEEE International Conference on, pp. 2100-2105, IEEE, 2013. 